\title{
Lasso Regularized Gabor Shearlet Face Multivariate Sparse Function Approximation
}

\author{
LI Dong-Rui ${ }^{1}$ \\ 1. Department of Computer, Guangdong AIB Polytechnic College, Guangzhou, \\ China \\ LDR_1983@126.com
}

\begin{abstract}
In allusion to such problems in the traditional face recognition methods as poor recognition accuracy and dissatisfactory processing effect for directivity and amisotropic characteristic in face data, lasso regularized Gabor sheaklet face nultivariate sparse function approximation algorithm is proposed in this article. Firstly, Gabor improved shearlet algorithm is adopted at the level of the face-tmage biologidal signals for the sparse expansion representation of the face ddta characteristics, and meanwhile this algorithm is also adopted to extract the geometrical characteristils of the expansion face with directivity and anisotropic characteristic. Secondly, in order to balance the algorithm effect, lasso regularization theory is introduced therein to control and weigh the relation between the fidelity and the smoothness of the face data. Finally, the corresponding simulation experimen is carried ont 10 compare the proposed algorithm and the existing algorithms in the standard testatabase in order to verify the advantages of the proposed algorithm in the dspect of face recognition accuracy and efficiency.
\end{abstract}

Keywords: Recognition accuracy; Shearlet; Face recognition; Smoothness; Sparse approximation

\section{Introduction}

As a classic research direction in the fields of computer vision and pattern recognition, face recognition is a biological recognition technology for identity recognition and tracking based on face characteristics. Currently, face recognition is widely applied m many fields, such as government, bank, military affairs, ecommerce, welfare guarantee and safety \& defense [1]. Actually, many achievement have been obtained for the research on face recognition algorithm, for example, E) genfaces recognition method proposed in literature [2], Fisher face diserimination method proposed in literature [3], support vector machine, neural netwo k, etc. The recognition subset selection is a standard statistical problem for face recognition and mainly includes the following operations ( $L_{0}$ regularization): $\hat{\omega} L_{0}=\arg \min _{\omega}\left[\|\mathbf{X} \boldsymbol{\omega}-\mathbf{y}\|_{2}^{2}\right]$, and this condition $\|\omega\|_{0} \leq \delta$ shall be met. In the above formula, $\delta$ is the setting parameter; $\mathbf{y}$ is the normalized face data; $\mathbf{X}$ is the face database matrix; $\omega$ is the weight matrix used for controlling and weighing the relation between the fidelity and the smoothness of the face data. This statistical method becomes a research hotspot for face recognition in recent years, because the sparse representation concept represented thereby has brought the possibility for the reconstruction of the face recognition data. For example, the sparse representation classification (SRC) scheme proposed in literature [4] regards the recognition problem as a multiple linear regression sparse classification problem and meanwhile $L_{1}$ is minimized to realize the effective extraction of the face 
characteristics; the image coding is searched for the sparse linear combination of all training samples, and the reconstruction error is minimized to realize the classification of the searched images. Even so, it is indicated in literature [5] that the success of SRC is ascribed to the coordination representation of the searched images rather than the sparse constrained code of $L_{1}$ norm. Besides SRC scheme, the regularized robust coding (RRC) method proposed in literature [6] can realize the robust regression of the regularization coefficient.

Actually, although the traditional wavelet transform is widely applied in image processing, yet it is restricted in the field of solving the information directivity problem [7]. In comparison, the shearlet can be used to provide more simple multiscale analysis framework and has higher efficiency for solving image directivity problem and anisotropic problem, thus to realize the optimum sparse representation and numeric rapid representation of $2 \mathrm{D} / 3 \mathrm{D}$ images. Lasso regularized Gabor shearlet face multivariate sparse function approximation solution is proposed in this article mainly on the basis of the sparse regularization theory. The main innovative presentation of this article is as follows: (1) efficient face characteristic extraction method based on direction characteristic; (2) the adoption of Lasso method for realizing regularization optimization based on the above extracted direction characteristic.

\section{Classification Algorithm Based onsparse Representation}

For $k$ clusters of training samples if the matrix composed of the $i$ th cluster of training samples is set as $\boldsymbol{D}_{i}=\left[\boldsymbol{d}_{i 1}, \boldsymbol{d}_{i 2}, \cdots, \boldsymbol{d}_{\mathrm{N} i}\right] \in R^{d \times n_{i}}$ ( $n_{i}$ is the number of the $i$ th cluster of training samples), then the whole training sample set can be expressed as $\boldsymbol{D}=\left[\boldsymbol{D}_{1}, \boldsymbol{D}_{2}, \cdots, \boldsymbol{D}_{k}\right] \in R^{d \times n}$, wherein $n$ refers to the total number of the training samples and is expressed as $n=\sum_{i=1}^{k} n_{i}$. If the training sample matrix $\boldsymbol{D}$ is regarded as a dictionary, then the given test sample $\boldsymbol{y}$ can be linearly expressed by dictionary $\boldsymbol{D}$, namely $\boldsymbol{y}=\boldsymbol{D} \boldsymbol{x}$ is believed in SRC that test sample $\boldsymbol{y}_{\text {can }}$ be fully linearly expressed by the similar training samples. Therefore, when there are enough training samples in training sample set $\boldsymbol{D}$, vector $\boldsymbol{x}$ of $\boldsymbol{y}$ in dictionary $\boldsymbol{D}$ is sparse.

In SRC, $l_{0}$ norm is replaced by $l_{1}$ norm to approximately represent the sparseness of vector $\boldsymbol{x}$, and meanwhile the noise influence is considered. Therefore, the sparsest solution of $\boldsymbol{y}=\boldsymbol{D} \boldsymbol{x}$ can be obtained through $l_{1}$ norm minimization problem. Specifically the optimization problem of $l_{1}$ norm can be expressed as follows:

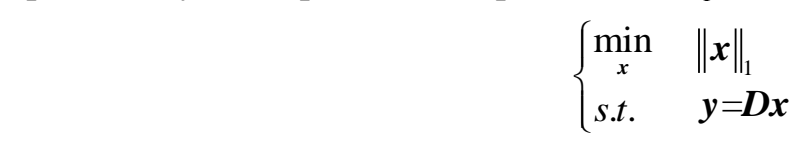

Minimization Problem (1) is a convex optimization problem and can be solved through rapid basis pursuit algorithm $[14,15]$.

On the basis of sparse representation coefficient $\hat{\boldsymbol{x}}$ obtained thereby, if $\delta_{i}(\hat{\boldsymbol{x}})$ is set as a vector which has the same dimension as $\hat{\boldsymbol{x}}$ and only reserves the nonzero elements corresponding to the $i$ th cluster in $\hat{\boldsymbol{x}}$ and in which other elements are zero, then approximate evaluation $\hat{\boldsymbol{y}}_{i}=\boldsymbol{D} \delta_{i}(\hat{\boldsymbol{x}})$ of the test sample correlated to the $i$ th cluster can be obtained. Further, the reconstruction error correlated to test sample $\boldsymbol{y}$ and each $i$ th cluster of training samples is obtained as follows:

$$
\boldsymbol{r}_{i}(\boldsymbol{y})=\left\|\boldsymbol{y}-\boldsymbol{D} \delta_{i}(\hat{\boldsymbol{x}})\right\|_{2}^{2}
$$


In SRC algorithm, the cluster with minimum reconstruction error $\boldsymbol{r}_{i}(\boldsymbol{y})$ is judged as the cluster containing test sample $\boldsymbol{y}$.

$$
\text { indentity }(\boldsymbol{y})=\arg \min _{i} \boldsymbol{r}_{i}(\boldsymbol{y})
$$

In the above formula, indentity $(\boldsymbol{y})$ denotes the cluster label of test sample $\boldsymbol{y}$.

In practical application, face images are usually polluted by noises and the image acquisition process may be influenced by such errors regarding face expression, posture, light condition, local obstruction, etc. For the above reason, unit matrix $I \in R^{d \times d}$ is introduced into SRC as the error dictionary to describe the face image noise and error. Then, the given test sample $\boldsymbol{y}_{\text {can }}$ be linearly and sparsely expressed by redundant dictionary $\boldsymbol{B}=[\boldsymbol{D} \boldsymbol{I}] \in R^{d \times(n+d)}$ composed of the training sample matrix and the error dictionary thereof. The specific formula is as follows:

$$
\begin{cases}\min _{\boldsymbol{w}} & \|\boldsymbol{w}\|_{1} \\ \text { s.t. } & \boldsymbol{y}=\boldsymbol{B} \boldsymbol{w}\end{cases}
$$

In the above formula, $\boldsymbol{w}=[\boldsymbol{x}, \boldsymbol{\beta}] ; \varepsilon$ is a positive constant. Similarly, the optimal solutions $\hat{\boldsymbol{x}}$ and $\hat{\boldsymbol{\beta}}$ can be obtained through solving Optimization Problem (4). Further, reconstruction error $\boldsymbol{r}_{i}(\boldsymbol{y})$ correlated to test sample $\boldsymbol{y}$ and each $i$ th cluster of training samples can be obtained, and $\boldsymbol{r}_{i}(\boldsymbol{y})$ is subsequently) adopted to classify the test sample according to Formula (3).

\section{Regularized Gabor Shearlet}

\subsection{Algorithm Framework}

Two-dimensional Gaboy wavelet transform is an important tool for signal analysis and processing in time-frequency domain, and the transform coefficient thereof has good visual characteristic and biological background. Gabor filters with different parameters can capture local characteristic information in the image, and such information is corresponding to different spatial frequencies, positions and directions. Due to the insensitivity to brightness and face expression change, Gabor filter is widely appled $1 \mathrm{n}$ such fields as image coding, handwritten number recognition, face recognition and edge detection.

Two-dimensional Gabor wavelet transform is adopted for the gray level image obtained after preprocessing in order to obtain the fatigue characteristics of the driver face area. Therein, the two-dimensional Gabor wavelet kernel function is as follows:

$$
\psi_{j}(\vec{k}, \vec{x})=\frac{\vec{k}_{j}^{2}}{\sigma^{2}} \exp \left(-\frac{\vec{k}_{j}^{2} \bullet \vec{x}^{2}}{2 \sigma^{2}}\right)\left[\exp \left(\vec{k}_{j}^{2} \bullet \vec{x}\right)-\exp \left(-\frac{\sigma^{2}}{2}\right)\right]
$$

In the above formula, $\vec{k}_{j}^{2}$ is adopted to ensure that the filters with different frequency bandwidths have approximate energy; $\exp \left(-\frac{\sigma^{2}}{2}\right)$ is adopted to compensate image DC shunt excitation. Obviously, the filters are insensitive to the overall lamination, thus to have the advantages of keeping the spatial relationship while describing the spatial frequency structure.

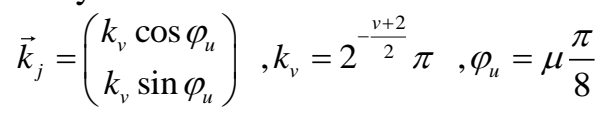

$\vec{k}_{j}$ forms different wavelets (with different edge values). Four dimensions $k_{v}=2^{-\frac{v+2}{2}} \pi(v=1,2,3,4)$, and six directions $\varphi: 0-6 / 8 \pi(\mu=1,2, \ldots, 6), \pi / 8$ are 
adopted in this article, wherein $\sigma$ interval is set as the filter length, namely $\sigma=\pi$, and the time frequency is set as 1 . If image $I(x, y)$ and convolution wavelet are input, then the following formula can be obtained:

$$
g\left(\vec{k}_{j}, \vec{x}\right)=\iint I(\vec{x}, \vec{y}) \varphi_{j}(\vec{k}, \vec{x}) d x d y
$$

In the above formula, $g\left(\vec{k}_{j}, \vec{x}\right)$ is the amplitude. Gabor filters have 48 characteristic points and accordingly form one group of optimum filters representing the object characteristics. The wavelet subspace is composed of these filters, and the image is projected to the wavelet subspace in order to obtain the wavelet coefficient. Afterwards, the mean value and the variance are extracted to represent the statistical characteristics of the driver face expression image.

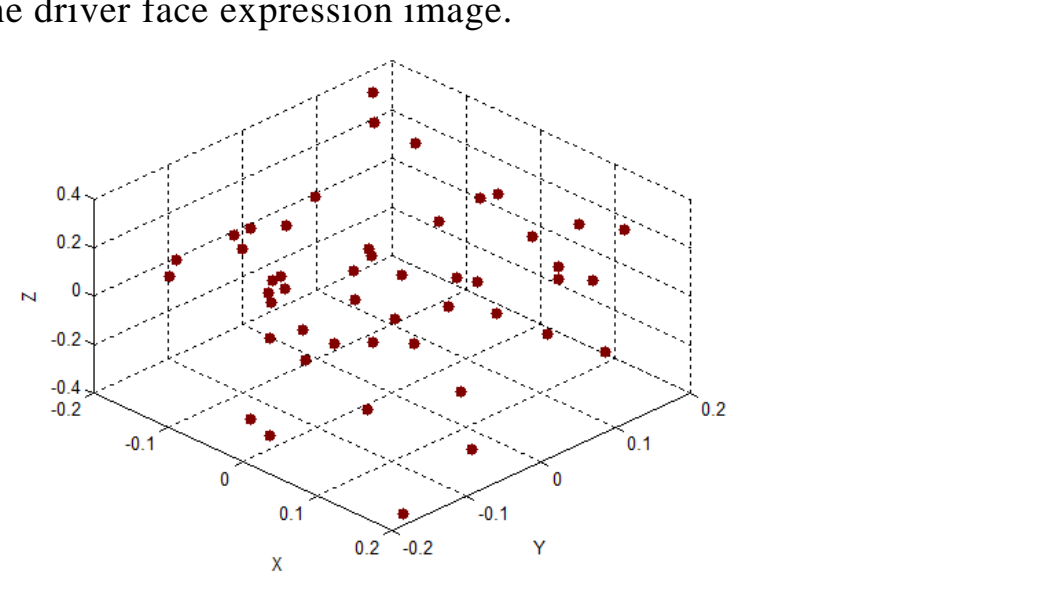

Figure 1. Distribution Diagram of 48 Characteristic Points

\subsection{Model Shearlet Characteristic Kxtraction}

For the regularized Gabor shelarlet method proposed in this article, the shearlet theory is firstly adopted to in tralize the face model. A compact biological signal can be generated through the shearlet network. The main feature of this method is as follows: the weight vatue is adopted for shearlet reconstruction in order to match with the individual images in the face database, as shown in Figure 2.

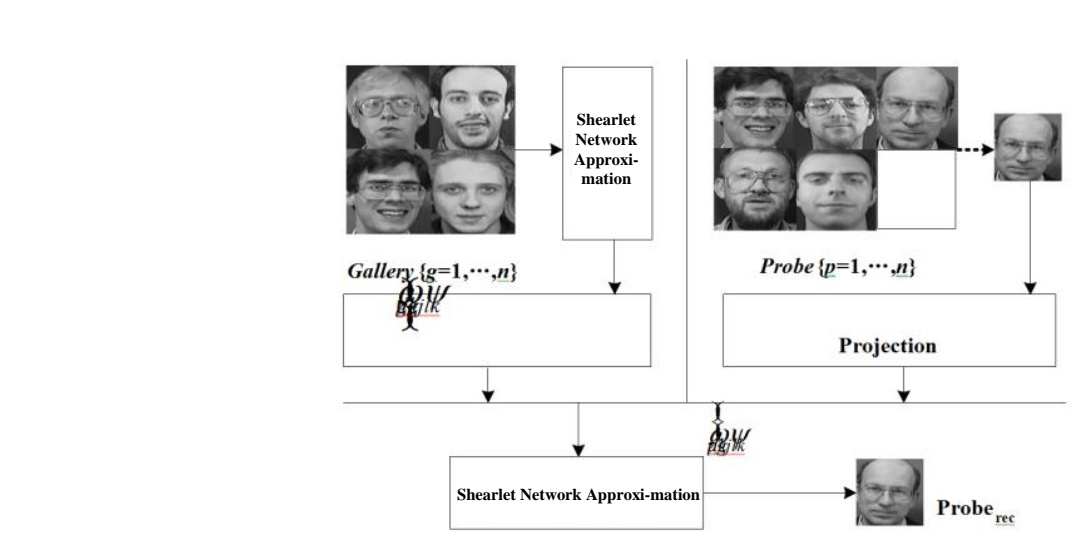

Figure 2. Shearlet Training Model

A strict framework can be formed through the shearlet, and this means that any spatial image can be represented through the following quadratically integrable function: 


$$
f=\sum_{j, k \in R, k \in R^{2}}\left\langle f, \psi_{j, l, k}\right\rangle \psi_{j, l, k}
$$

The above formula is adopted to define the shearlet algorithm, similarly to hybrid RBF neural network and the wavelet network decomposed by shearlet. In the optimization stage, the weight connection of each stage can be calculated and analyzed through mapping the signal to the shearlet. Specifically, the model formula is as follows:

$$
\left\{\begin{array}{l}
\tilde{\psi}_{j, l, k}^{t}=\sum_{m=1}^{N}\left(\psi_{i, m}\right)^{-1} \psi_{j, l, k}^{m} \\
\psi_{i, m}=\left\langle\psi_{j, l, k}^{i}, \psi_{j, l, k}^{m}\right\rangle
\end{array}\right.
$$

\subsection{Description of the Proposed Algorithm}

$\left(e_{i n i t}\right)_{l}$ denotes the $l$ th maximum element in set $e_{\text {init }}^{2}(j), j=1, \cdots, n$, and can be calculated according to the following formula:

$$
e_{\text {init }}=\|\mathbf{y}-\operatorname{mexh}(\mathbf{X})\|^{2}
$$

In the above formula, $\mathbf{X}$ as the face data is a matrix with the size of $n \times d ; \mathbf{y}$ as the normalized face test image is the vector data with the size of $n \times 1$. After optimization, the following formula can be adopted to calculate rest energy $e$ :

$$
\text { e }=\left(\mathbf{X} \omega_{i}-\mathbf{y}\right)^{2}
$$

In the above formula, $\omega_{i}$ is the Aweight value obtained after Lasso optimization ( $L_{1}$ regularization theory). Then, thle minimum cluster of sample $\mathbf{y}$ is searched for face recognition, and the judgment standard is as follows:

$$
\operatorname{ern} \operatorname{dr}(y)=\left\|\omega^{\frac{1}{2}}(\mathbf{y}-\mathbf{X} \omega)\right\|_{2}^{2}
$$

In the above formula, $\mathbf{X}$ is the face image reconstructed through the shearlet characteristic extraction of the model; $\mathbf{y}$ is the test image reconstructed through projection and characteristic extraction; $\omega$ is the weight value. The process is as shown in Figure 3. The steps of the regularized Gabor shearlet face recognition algorithm are as shownun Pseudo Code 1.

Pseudo Code 1 Regularized Gabor Shearlet Face Recognition

Input:

Normalized face test image; $\mathbf{f}: \mathbf{y}=\mathbf{f} / \operatorname{norm}(\mathbf{f}, 2)$;

$\mathbf{X}$ : Face database ordering; $\mathbf{X}=\mathbf{X} / \sum x \cdot x$;

-- Iter: Maximum iteration number;

$$
\begin{aligned}
& -\boldsymbol{\omega} ; \operatorname{identity}(y) ; \\
& ---\boldsymbol{\omega} \text { : identity }(y)
\end{aligned}
$$

Algorithm Steps:

1. Select $\omega_{\text {init }}$ according to Formula (12);

2. Diagonalize matrix $\mathbf{X}, \mathbf{X}^{t}=\mathbf{X}^{\prime} \cdot \mathbf{X} ; \mathbf{y}^{t}=\mathbf{X}^{\prime} \cdot \mathbf{y}$

3. for $i=\mathbf{1}$ : Iter do

4. Calculate according to Formula (8): 


$$
\begin{aligned}
& \text { 5. } \quad \hat{\omega} L_{1}=\arg \min _{\omega}\left[\frac{1}{n}\|\mathbf{X} \boldsymbol{\omega}-\mathbf{y}\|_{2}^{2}+\lambda\|\boldsymbol{\omega}\|_{1}\right] \\
& \text { 6. } \omega_{i}=\text { lasso }\left(\mathbf{X}, \mathbf{y}, \mathbf{X}^{t}, \mathbf{y}^{t}, \omega_{\text {init }}, \omega_{\text {thre }}\right) ; \\
& \text { 7. } \quad \omega_{\text {init }}=\omega_{i} ; \\
& \text { 8. endfor } \\
& \text { 9. } y_{\text {rec }}=\mathbf{X} \cdot \omega_{i} ; \omega=\omega_{i} ; \\
& \text { 10. if } k=1 \text { do }
\end{aligned}
$$

11. $\operatorname{error}(y)=\left\|\omega^{1 / 2}(\mathbf{y}-\mathbf{X} \omega)\right\|_{2}^{2}$;

12. endif

13. identity $(y)=\arg \min _{h}($ error $)$;

\section{Experiment and Analysis}

\subsection{Experiment Conditions}

Face recognition is an extremely challenging research subject in the fields of computer vision and pattern recognition. Four large face databases are mainly adopted for this experiment: ORL, YALE, FERET and DCSKU. Specifically, ORL database includes ten different image combinations of 40 different subjects. Due to the photographing difference, the images photographed by different persons are different from each other in the aspects of light condition, face expression and face details. Additionally, Yale database includes 1,265 gray level images in GIF format for 15 persons; FERET database include 1,196 front face images; DCSKU database includes 1,000 face images photographed under different conditions for 100 men and 50 women. $2 / 3$ of the images are selected as the training images and $1 / 3$ of the images are selected ${ }^{\circ}$ as the test images

In this experiment, all face images shall be normalized into the images with the size of $128 \times 128$. The hardware condition of the experiment is as follows: processor CPU: Inter(R) Oore(TM) 33-3110 @2.40GHz; memory RAM: ddr3 1600 4G. Some images in the above databases are as shown in Figure 3.

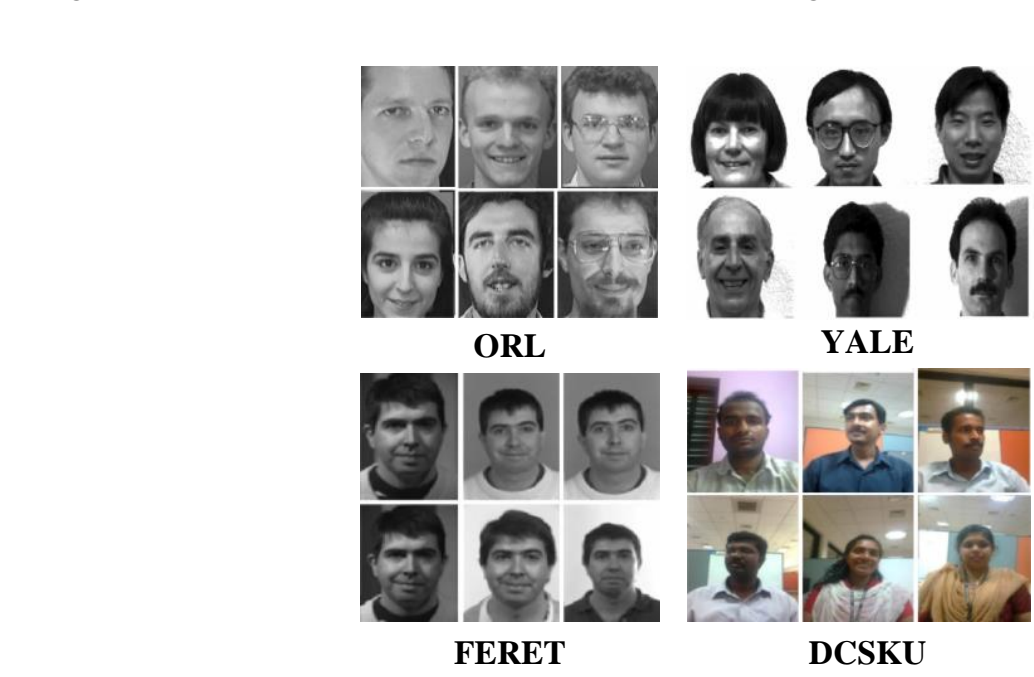

Figure 3. Face Database 


\subsection{Recognition Accuracy}

As mentioned above, the size of the normalized image is selected as $128 \times 128$, and the frequency of Gabor filter is selected as $f_{\max }=\pi / 2$. Eigenface algorithm, Fisherface algorithm, SRC algorithm and LGBPHS [11] algorithm mentioned above are selected as the comparison algorithms. The recognition accuracy is selected as the evaluation index and can be defined as follows:

$$
R A=1-E E R
$$

In the above formula, $E E R$ is the equal error rate index.

The evaluation indexes of LRGS algorithm proposed in this article and the comparison algorithms are compared in the test face database. The comparison results are as shown in Tab.1, and the average run time of the algorithms is as shown in Tab.2 (the average run time obtained through running each algorithm for 20times).

Table 1. Comparison Results of Recognition Accuracy

\begin{tabular}{ccccccc}
\hline Database/Algorithm & Eigenface & Fisherface & LGBPHS & SRC & LRGS \\
\hline ORL & 0.5921 & 0.8450 & 0.8737 & 0.1519 & 0.9600 \\
YALE & 0.6053 & 0.8750 & 0.8637 & 0.7256 & 0.9600 \\
FERET & 0.5697 & 0.7500 & 0.8187 & 0.7165 & 0.9250 \\
DCSKU & 0.6842 & 0.8250 & 0.8837 & 0.7627 & 0.9698 \\
\hline
\end{tabular}

The simulation comparison resuft of the four comparison algorithms in such face databases as ORL are as shown in Tab.2. From the aspect of the recognition accuracy index, the recognition accuracies of Eigenface algorithm, Fisherface algorithm, LGBPHS algøithm, SRC algorithms and the proposed algorithm are respectively in the ranges of $56 \%-69 \%, 75 \% \sim 85 \%, 81 \% \sim 89 \%, 71 \% \sim 77 \%$ and 92\% 97\%. Obvioushy LRGS algorithm is superior to such comparison algorithms as Eigenface algorithm in the aspect of recognition accuracy.

The run time of the four compatison algorithms in such face databases as ORL is as shown in Tab.3. According to the simulation results, the run time of LRGS algorithm in ORL face database is $2.516 \mathrm{~s}$, longer than that of Fisherface algorithm but shorter than that of Eigenface algorithm and LGBPHS algorithm; LRGS algorithm has the fastest run speed in YALE database; the run time of LRGS algorithm in FERET database is longer than that of Eigenface algorithm and LGBPHS aldorithm but shorter than that of Fisherface algorithm; LRGS algorithm also has fastest run speed in DCSKU database; SRC algorithm has moderate or slower run speed in the above face databases. In conclusion, LRGS algorithm has optimym run time in the above face databases.

Table 2. Comparison of the Average Run Time

\begin{tabular}{cccccc}
\hline Database/Algorithm & Eigenface & Fisherface & LGBPHS & SRC & LRGS \\
\hline ORL & 6.415 & 2.133 & 3.146 & 4.653 & 2.516 \\
YALE & 5.042 & 6.500 & 5.238 & 4.814 & 3.325 \\
FERET & 4.610 & 5.113 & 3.473 & 5.986 & 4.786 \\
DCSKU & 5.867 & 3.102 & 4.443 & 3.846 & 2.758 \\
\hline
\end{tabular}




\section{Conclusion}

Gabor shearlet face multivariate sparse function approximation algorithm based on Lasso regularization is proposed in this article to effectively solve such problems in traditional face recognition as poor algorithm accuracy and dissatisfactory processing effects for directivity and anisotropic characteristic in face data. According to the experiment comparison in four standard face databases, namely ORL, YALE, FERET and DCSKU, LRGS algorithm proposed in this article is superior to Eigenface algorithm, Fisherface algorithm, SRC algorithm and LGBPHS algorithm in the aspects of recognition accuracy and run time. Meanwhile, the selection comparison results of the normalized image size and Gabor filter frequency are also given in this experiment in order to provide reference for selecting algorithm parameters.

\section{References}

[1] Z. Lv, L. Feng, S. Feng and H. Li. "Extending Touch-less Interdction on Visłon Based Wearable Device", Virtual Reality (VR), 2015 iEEE, IEEE, (2015).

[2] M. Zhang, Z. Lv, X. Zhang, G. Chen and K.Zhang. "Research and Application of the 3D Virtual Community Based on WEBVR and RIA", Computer and Information Science, vol. 2, no. 1 (2009), pp84.

[3] J. He, Y. Geng and K. Pahlavan, "Modeling Indoor TOA Ranging Error for Body Mounted Sensors", 2012 IEEE 23nd International Symposium on Personal Indoor and Mobile Radio Communications (PIMRC), Sydney, Australia, (2012), pp. 682-686

[4] Y. Geng, J. Chen and K. Pahlavan, "Motion detection using RP signals for the first responder in emergency operations: A PHASER project" 2013 IEEE 24nd International Symposium on Personal Indoor and Mobile Radio Communications (PMRC) London, Britain, (2013).

[5] S. Li, Y. Geng, J. He and K/ Phlavan, "Analysis of Three-dimensional Maximum Likelihood Algorithm for Capsule Endoscopy Localization", 2012 5th International Conference on Biomedical Engineering and Informatics (BMEl), Chongqing, China, (2012), pp. 721-725.

[6] Y. Geng, J. He, H. Deng and K. Pahlavan, "Modehing the Effect of Human Body on TOA Ranging for Indoor Human Tracking (vitb) Wrist Mounted Sensor", 16th International Symposium on Wireless Personal Multimedia Communications (WPMC), Atlantic City, NJ, (2013).

[7] Y. Geng, J. He and K. Păhlavan, "Modeling the Effect of Human Body on TOA Based Indoor Human Tracking", International Journal of Wineless Information Networks, vol. 20, no. 4, pp. 306-317.

[8] T. Su, Z. LV, S. Gao, X. Li and H. Lv, "3D seabed: 3D modeling and visualization platform for the seabed”, In Multimedia andExpo Workshops (ICMEW), 2014 IEEE International Conference on, IEEE, (2014), pp. 1-6.

[9] X. Li, Z. Lv, B. Zhang, W. Wang, S. Feng and Ji. Hu, "WebVRGIS Based City Bigdata 3D Visualization and Analysis", In Pacific Visualization Symposium (PacificVis), 2015 IEEE. IEEE, (2015).

[10] X. Li, Z. Lv, B. Zhang, L. Yin, W. Wang, S. Feng and J. Hu, "Traffic Management and Forecasting System/Based on 3D GIS", Cluster, Cloud and Grid Computing (CCGrid), 2015 15th IEEE/ACM International Symposium on. IEEE, (2015).

[11] X. Li, Z. Lv, J. Hu, B. Zhang, L.Y.Shi and S. Feng, "XEarth: A 3D GIS Platform for managing massive cit information", IEEE Computational Intelligence and Virtual Environments for Measurement Systems and Applications (CIVEMSA), IEEE, (2015).

[12] A. Tek, B. Laurent, M. Piuzzi, Z. Lu, M. Chavent, M. Baaden and O. Delalandel, "Advances in HumanProtein Interaction-Interactive and Immersive Molecular Simulations", InTech, (2012).

[13] Zhong, Chen, S. M. Arisona, X. Huang, M. Batty and G. Schmitt, "Detecting the dynamics of urban structure through spatial network analysis", International Journal of Geographical Information Science, vol. 28, no. 11, (2014), pp. 2178-2199.

[14] R. Ma, Z. Lv, Y. Han and G. Chen, "Research and Implementation of Geocoding Searching and Lambert Projection Transformation Based on WebGIS”, Geospatial Information, vol. 5, (2009), pp. 013. 


\section{Author}

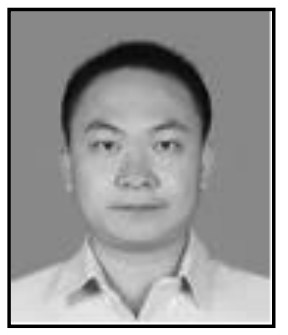

LI Dong-rui, He was born in Guangxi province of China in 1983. He has received his bachelor degree and master degree respectively. Currently, he is a lecturer. His research interests include Pattern Recognition and Image Processing.

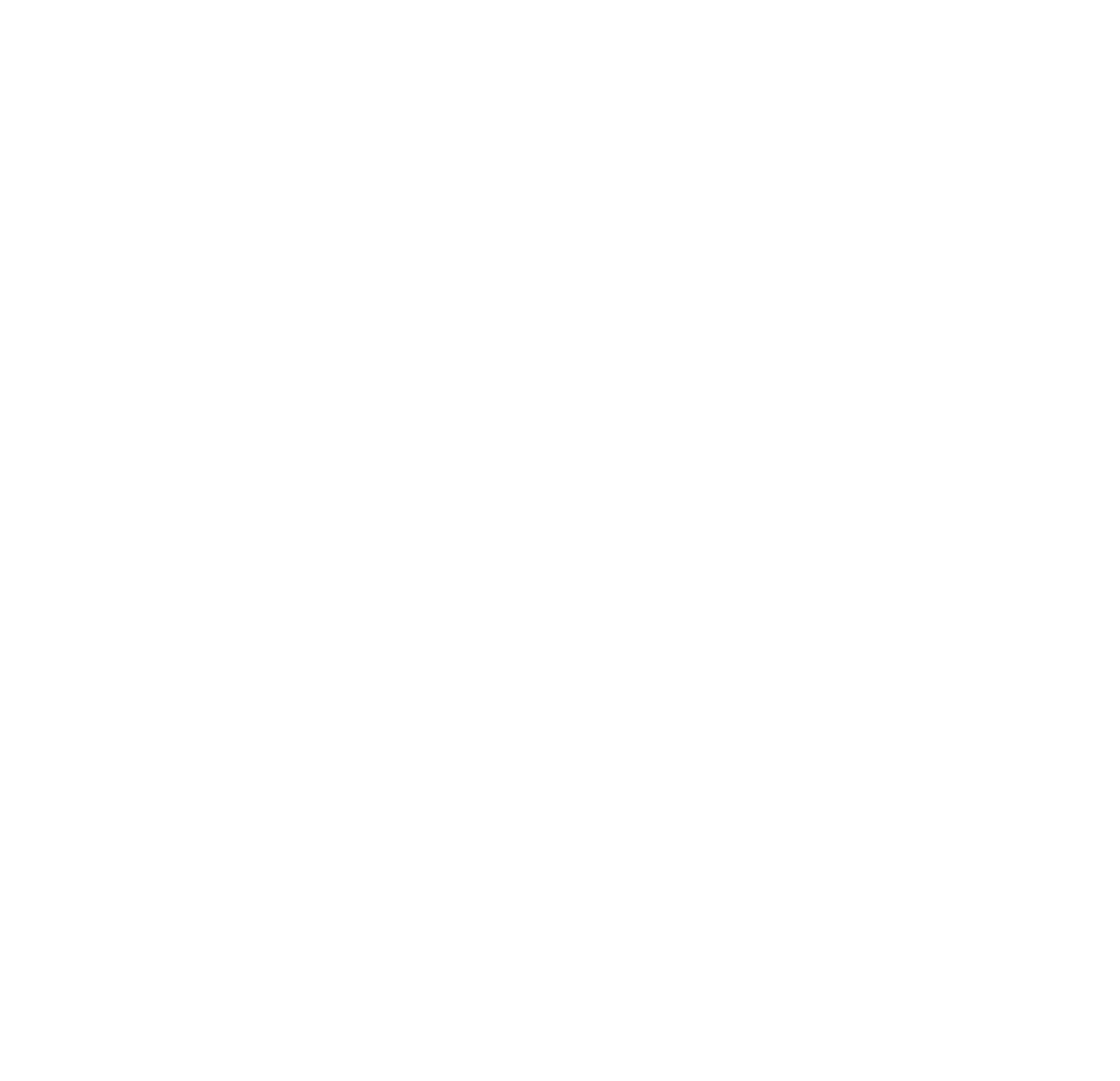


International Journal of Hybrid Information Technology

Vol. 9, No.8 (2016)

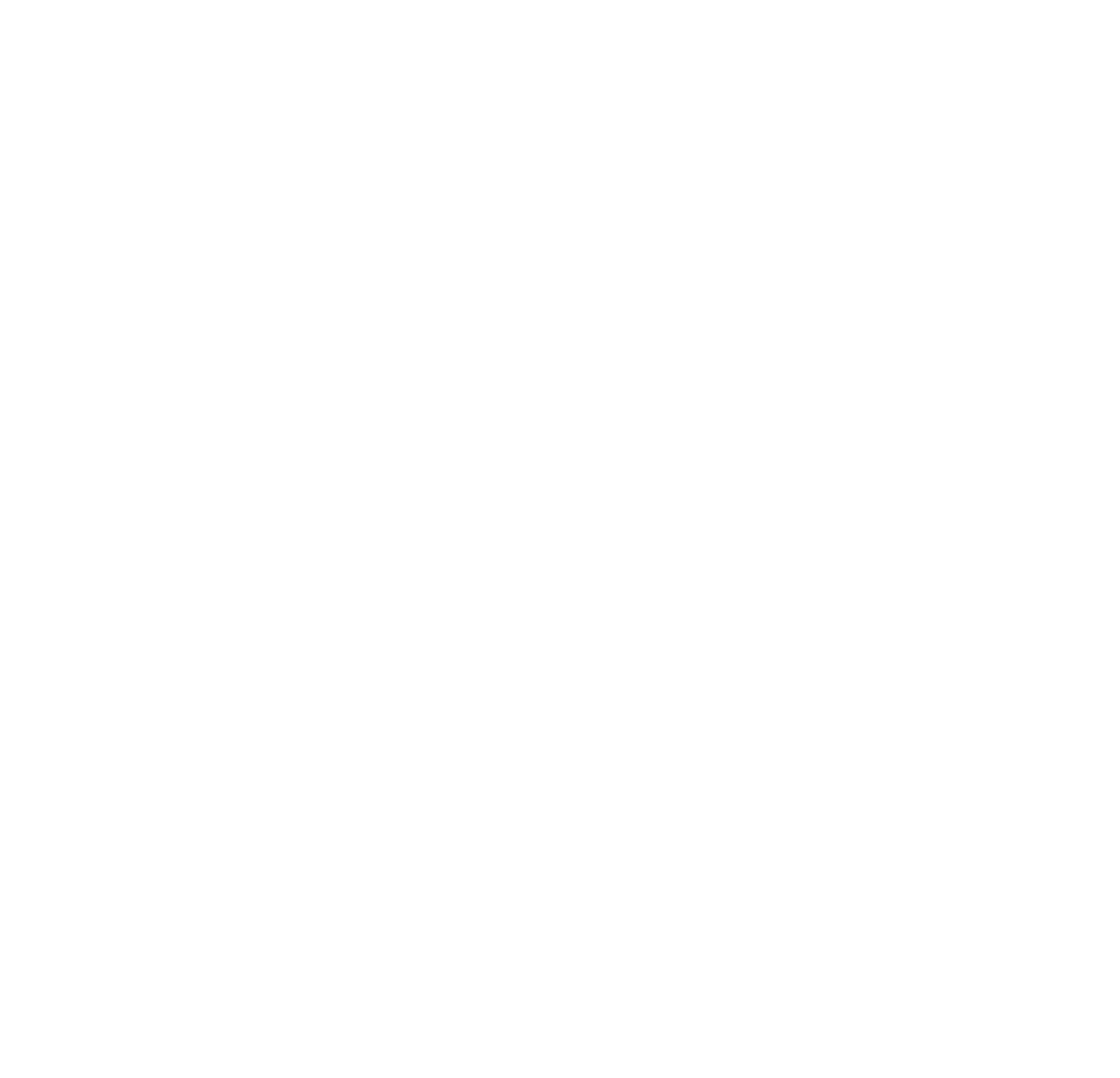

